\title{
Effect of lesimarin against thioacetamide-induced liver cirrhosis in rat
}

\author{
Sang-Ho Ra ${ }^{\oplus 1}$, Ri-Hwa Shin ${ }^{1}$, Hak-Chol Ri ${ }^{1 *}$, Jong-Hui Ri ${ }^{1}$, Hui-Chol Ri ${ }^{1}$, Ae-Jong Ri ${ }^{1}$ \\ ${ }^{1}$ Department of Pharmacology, Basic Medical Faculty, Pyongyang Medical College, Kim Il Sung University, \\ Central District, Pyongyang, Democratic People's Republic of Korea
}

\begin{abstract}
Liver cirrhosis is one of chronic liver diseases with high disability and mortality accompanying fibrosis, hepatocyte damage, and liver dysfunction. In this study, the hepatoprotective and the antifibrotic properties of lesimarin(lecithin - silymarin - Artemisia messerschmitiana Besser(AMB) extract complex at 11:3:6 ratio) on rat hepatic fibrosis induced by thioacetamide (TAA) was investigated. Rats were divided into seven groups: control, lesimarin, TAA, TAA+lesimarin, TAA+lecithin, TAA+silymarin, TAA+AMB. Rats were administered with TAA at a dose of $200 \mathrm{mg} / \mathrm{kg}$ body weight intraperitoneally twice a week for three months. Lesimarin, lecithin, silymarin and AMB were administered at a dose of $1.0,1.0,0.5,1.0 \mathrm{~g} / \mathrm{kg}$ body weight orally daily for three months, respectively. TAA administration resulted in hepatic fibrosis, significant decrease in body weight, albumin level and A/G ratio and increase in plasma transaminase, GGT( $\gamma$-glutamyltransferase) and ALP(alkaline phosphatase) activities as well as hepatic hydroxyproline content, which were attenuated by lesimarin administration. Lesimarin was found to decrease AST, ALT and GGT, ALP and bilirubin, hydroxyproline levels and increase albumin level and A/G ratio and its effect is more prominent than those of individual constituents. These results suggest this new drug, lesimarin, might be a promising drug to be used for chronic liver diseases.
\end{abstract}

Keywords: Antifibrotic effect. Lesimarin. Thioacetamide. Hepatic fibrosis.

\section{INTRODUCTION}

The liver disorders are very frequent and widely distributed in the world. Among them hepatic fibrosis represents the response of the liver to different chronic insults, and is associated with significant morbidity and mortality. Recent studies have clearly demonstrated that the excessive accumulation of extracellular matrix in liver fibrotic diseases is a dynamic and bidirectional process which is mainly regulated by hepatic stellate cells (HSC) (Abul et al., 2002). Several in vitro studies have shown that the process of HSC activation results from the complex interplay of different factors, such as cytokines, growth factors, oxidative stress and modification of $\mathrm{Na}^{+} / \mathrm{H}^{+}$exchange activity (Aydin et al., 2010).

In several experimental models for the induction of cirrhosis, hepatotoxic agents such as thioacetamide (TAA), carbon tetrachloride and ethionine have been

\footnotetext{
*Correspondence: H.-C. Ri. Department of Pharmacology, Basic Medical Faculty, Pyongyang Medical College, Kim Il Sung University, Central District, Pyongyang, Democratic People's Republic of Korea. E-mail: ryongnam17@yahoo.com
}

used. Thioacetamide (TAA) has been widely used for experimentally induced liver injury. While acute TAA application results in hepatitis, chronic TAA application has been shown to cause liver cirrhosis (Chilakapati et al., 2007). Oxidative stress has been reported to play an important role in the pathogenesis of TAA-induced hepatitis and cirrhosis. Indeed, several studies have demonstrated the beneficial effect of antioxidants in protecting the liver against TAA-induced injury (Palacios et al., 2008).

Silymarin extracted from the milk thistle is well regarded for its antioxidant and protective effects on the liver. It has been shown to protect the liver against increased oxidative stress caused by alcohol consumption, $\mathrm{CCl}_{4}$ or TAA and accelerate alcohol metabolism (Chen et al., 2012; Chung-His et al., 2012).

The elevated levels of liver enzymes such as aspartate aminotransferase (AST) and alanine aminotransferase (ALT) found in liver injuries and chronic diseases are reduced significantly (30-35\%) by its use. Silymarin has been shown to reduce liver fibrosis up to $30-35 \%$, and in few cases it has reversed the liver fibrosis. It has also been 
Sang-Ho R., Ri-Hwa S., Hak-Chol R., Jong-Hui R., Hui-Chol R., Ae-Jong R.

reported to have anti-inflammatory, immunomodulatory, antiviral and liver-regenerating properties (Adeyemo et al., 2013; Freedman et al., 2011; Mahmood et al., 2014). It also causes an improvement of liver steatosis and inflammation by decreasing membrane lipid peroxidation, reducing free-radical release and restoration in the GSH levels (Carina et al., 2012). In addition, it was found to induce reduction of leukotriene formation from unsaturated free acid, enhance protein synthesis, stabilize mast cell, inhibit cytP450 detoxification system and prevent metabolism of toxic compound such as thioacetamide, carbon tetracholoride and acetaminophen (Madani et al., 2008).

Lecithin is a group of yellow brownish fatty substances occurring in animal and plant tissues, and in egg yolk (Korbanjhon, Chungang, 2013; Carina et al., 2013). Lecithin is the major component of all the cell membranes and plays an important role in the normal function of the cells. It was found that lecithin stimulated bile lipid secretion and prevented a cholestatic effect of cyclosporine A -induced reduction of bile flow and an alteration of F-actin microfilaments in the liver. And its effect might be related to the effect of dietary lipids on chemical and physical composition of the hepatocyte plasma membrane (Benkoel et al., 2000). On the other hand, lecithin is known to prevent fatty liver by ensuring triglycerides export out of the liver and as such can be considered as promising therapeutic agents in the realm of functional foods (Maida, Sugas, Marica, 2012).

Artemisia messerschmidtiana Besser(AMB) is a traditional herb, which contains various kinds of active ingredients such as scopoletin, chlorogenic acid, volatile oil and $\beta$-sitosterol etc. These substances have cholagogic, antibacterial, diuretic and hemostatic actions and are effective against various organic liver diseases such as fatty liver and hepatic necrosis. In addition, it was found to increase amount of bile both in the normal rat and rat model of liver injury via carbon tetrachloride $\left(\mathrm{CCl}_{4}\right)$ and to decrease serum transaminase activities (Konovalova, Rybalko, 1977). Therefore, it has been traditionally used in the treatment of various liver dysfunction in several Asian countries including our country.

Although a lot of modern medicines are developed, discovering a new drug for treating various liver diseases remained still as a difficult problem. The pharmacological effects of above mentioned individual constituents have been reported, but there is no scientific evidence regarding the safety and efficacy of them in combination.

The purpose of the present study was to evaluate the hepatoprotective and the antifibrotic effects of a new drug, Lesimarin(mixture of lecithin, silymarin and Artemisia messerschmitiana Besser extract at 11:3:6 ratio).

\section{MATERIAL AND METHODS}

\section{Material}

TAA and silymarin was purchased from Sigma Aldrich (St Louis, MO, USA) and dissolved in sterile saline until all the crystals were dissolved. Lesimarin was the product of new technical developmental institute, Mannyon pharmaceutical company, DPRK. It was made by mixing lecithin, silymarin and Artemisia messerschmitiana Besser extract at 11:3:6 ratio. Lecithin was obtained from Sangon (Shanghai, China) Artemisia messerschmitiana Besser collected in suburb of Pyongyang was decocted until its humidity is $40 \%$ and used in the experiment. $\mathrm{HCl}$ were obtained from Sigma Aldrich (St. Louis, USA).

\section{Experimental animals and chronic administrations of Lesimarin}

Wistar rats, aged 8weeks, were obtained from the Animal Center of Pyongyang Medical College, Kim Il Sung University. The animals were allowed free access to food and water and were kept in wire-bottomed stainless steel cages. They were maintained in a temperaturecontrolled room $\left(25 \pm 3{ }^{\circ} \mathrm{C}\right)$ and kept on a $12 \mathrm{~h} / 12 \mathrm{~h}$ light/ dark. Eighty four animals were divided into seven groups: control, Lesimarin(LES), TAA, TAA+Lesimarin, TAA+lecithin(LEC), TAA+silymarin(SIL), TAA + AMB. Control group received standard laboratory chow. TAA group received $200 \mathrm{mg}$ TAA/kg body weight intraperitoneally twice a week for three months as in a previous paper (Aydin et al., 2010). Lesimarin, lecithin, silymarin and AMB were administered to the rats at a dose of $1.0,1.0,0.5,1.0 \mathrm{~g} / \mathrm{kg}$ body weight orally daily for three months, respectively. At the end of three months, rats were fasted overnight and killed by collecting blood into heparinized tubes by cardiac puncture, after which analysis of serum biochemistry and liver histopathology was performed to confirm chronic liver fibrosis and the effect of mixture.

\section{Isolation of specimens and determination}

Body weight, liver size, serum biochemical values and histopathological results of the rats in all the groups were analysed at the end of the 12 week experiment. The body weights of the animals were recorded weekly beginning from day 1 and throughout to the end of the experiment. The livers of the rats were isolated, dissected, and then rinsed in normal saline. Following this, the liver was blotted with filter paper and weighed. 
The liver index was calculated as the percentage of the body weight.

Blood samples were obtained by intracardiac puncture. Serum was separated from blood samples by centrifugation at $3000 \times g$ for $10 \mathrm{~min}$. Activities of aspartate aminotransferase (AST), alanine aminotransferase (ALT), $\gamma$-glutamyl transpeptidase (GGT), alkaline phosphatase (ALP) and total protein(T-protein) were determined using commercial enzymatic kits with a SPOTCHEM ${ }^{\mathrm{TM}} \mathrm{EZ}$ SP-4430 biochemistry analyser (ARKRAY, Inc., Kyoto, Japan), as described previously (Chen et al., 2012).

Liver hydroxyproline content was determined by using liver specimens (100-250 mg) which were homogenized in buffer and hydrolyzed in $2 \mathrm{~mL} 6 \mathrm{~N} \mathrm{HCl}$ at $110{ }^{\circ} \mathrm{C}$ for $24 \mathrm{~h}$. After that the hydrolysis samples were treated and quantified spectrophotometrically at $565 \mathrm{~nm}$ using a colorimetric analyser (Dri-Chem 3000, Fuji Photo Film Co, Tokyo, Japan) (Switzer et al., 1991).

\section{Histopathological analysis}

Hepatic morphology was assessed by light microscopy. Liver tissue (i.e. $1 \mathrm{~cm} \times 1 \mathrm{~cm} \times 0.5 \mathrm{~cm}$ ) was taken from the largest right lobe of the liver and fixed in $10 \%$ of formalin solution for 1 week. The liver tissue was dehydrated using a sequence of ethanol solutions, embedded in paraffin and sectioned to $5 \mu \mathrm{m}$ in thickness at $50 \mathrm{~mm}$ intervals followed by staining with hematoxylineosin (HE) dye. Masson's trichrome stain was used for the observation of liver fibrosis. (Shaker et al., 2011).

\section{Statistical analysis}

All results are expressed as mean $\pm \mathrm{SD}$ (standard deviation). Differences between other groups were tested by using analysis of variance (ANOVA). Where significant effects were found, T-test was performed. $\mathrm{p}<0.05$ was considered to be significant.

\section{RESULTS AND DISCUSSION}

Liver fibrosis represents a major medical problem with significant morbidity and mortality. It is the common response to chronic liver injury, ultimately leading to cirrhosis and its complications, portal hypertension, liver failure, and hepatocellular carcinoma (Bataller, Brenner, 2005). TAA-induced liver fibrosis is an ideal model to test potential antifibrotic drugs because the hepatotoxin TAA is well known to produce a hardly reversible fibrosis in rodents similar to that of humans (Laleman, Vander, Zeegers, 2006).

In the present study, there was a statistically significant reduction of body weight in rats administered with TAA compared to the normal and lesimarin-treated groups. This reduction in body weight could strongly be attributed to the toxic effect of TAA throughout the period of the experiment. This is considered to be the most reliable and consistent symptoms of toxicity among the experimental animals (Ljubuncic et al., 2005). Although there was an increase in body weight in TAA+LES, TAA+LEC, TAA+SIL, $\mathrm{TAA}+\mathrm{AMB}$ group compared with TAA group, but there was no significant difference between them. Concurrently, the liver to body weight ratio showed a corresponding significant difference between all treated groups compared to TAA group. The increase in body weight of rats treated only with Lesimarin is similar to that of the normal control group. The result revealed that Lesimarin has a tendency to normalize the liver function (Table I).

Our experimental data also showed a damage of liver tissue during TAA-administration for 12 weeks.

TAA-induced damage of liver can be linked to TAA-metabolism by hepatic CYP2E1 to TAA-sulfoxide,

TABLE I - Body weight and liver weights of the rats in different groups

\begin{tabular}{lccc}
\hline Group & Body weight $(\mathbf{g})$ & Liver weight $(\mathbf{g})$ & Liver index (\%) \\
\hline Control & $217.80 \pm 13.68^{*}$ & $6.85 \pm 0.73^{*}$ & $3.18 \pm 0.41^{*}$ \\
LES & $223.51 \pm 13.59^{*}$ & $6.91 \pm 0.71^{*}$ & $3.09 \pm 0.37^{*}$ \\
TAA & $175.32 \pm 7.51^{\#}$ & $8.25 \pm 0.95^{*}$ & $4.75 \pm 0.56^{\#}$ \\
TAA+LES & $202.24 \pm 12.53$ & $7.03 \pm 0.81$ & $3.36 \pm 0.45^{*}$ \\
TAA+LEC & $189.36 \pm 10.32$ & $7.20 \pm 0.76$ & $3.75 \pm 0.48^{*}$ \\
TAA+SIL & $198.75 \pm 12.24$ & $7.06 \pm 0.82$ & $3.63 \pm 0.28^{*}$ \\
TAA+AMB & $184.54 \pm 9.87$ & $7.32 \pm 0.83$ & $3.86 \pm 0.51^{*}$ \\
\hline
\end{tabular}

The data were stated as mean \pm SD. Means with different superscripts are significantly different. $* P<0.05$ versus TAA group and ${ }^{*} P<0.05$ versus TAA+LES group. LES stands for Lesimarin. TAA stands for thioacetamide. LEC, SIL, AMB stand for lecithin, silymarin, Artemisia messerschmitiana Besser, respectively. 
Sang-Ho R., Ri-Hwa S., Hak-Chol R., Jong-Hui R., Hui-Chol R., Ae-Jong R.

and to the highly reactive TAA-sulfdioxide that is responsible for TAA-induced hepatotoxic effects. During TAA-metabolism, both flavin-containing monooxygenase and cytochrome $\mathrm{P} 450$ reduce dioxygen to $\mathrm{O}_{2} \cdot$, which is catalyzed to form $\mathrm{H}_{2} \mathrm{O}_{2}$. $\mathrm{H}_{2} \mathrm{O}_{2}$ may generate the highly reactive hydroxyl radical $(\cdot \mathrm{OH})$ by the Fenton reaction, which is responsible for enhanced lipid peroxidation (Shaker et al., 2011).

In the present study, we found that a 12 -week regimen of Lesimarin can improve the symptoms of hepatic fibrosis induced by TAA. All drug-treated groups showed significant decrease of plasma ALT and AST levels compared with TAA-treated rats, but the effects of other individual constituents of mixture are not so potent as that of Lesimarin indicating the benefit of new drug (Table II). Enzymes such as ALT and AST are often used as biochemical markers of hepatic injury. In damaged liver cells, the ALT and AST leak into the blood stream, leading to an increase in the plasma levels. Thus elevation of plasma AST and ALT activities in the serum could be regarded as an index of damage of the liver parenchyma cells (Ching Yang et al., 2013). Lesimarin lowered GGT, ALP, total bilirubin level significantly compared with TAA-group (Table III). The toxic effect of TAA was also alleviated by the individual constituents of mixture, of which extents are inferior to lesimarin. GGT, ALP activities indicate the function of epithelial cells of biliary duct and it is elevated in the case of biliary tract diseases. Total bilirubin level also shows the function of hepatic cells and the state of bile excretion. These results suggest that Lesimarin could improve the bile duct function.

Albumin level and $\mathrm{A} / \mathrm{G}$ ratio were increased in all drug-treated groups compared with TAA-treated group, but there was no difference in total protein level between all treated groups (Table IV). And TAA+LES group
TABLE II - Effect of TAA, Lesimarin and its constituents on AST, ALT activities

\begin{tabular}{lcc}
\hline Group & ALT (U/L) & AST (U/L) \\
\hline Control & $43.2 \pm 6.4^{* \#}$ & $104.4 \pm 15.9^{* \#}$ \\
LES & $41.3 \pm 5.7^{* \#}$ & $99.5 \pm 12.7^{* \#}$ \\
TAA & $135.2 \pm 16.6^{\#}$ & $216.4 \pm 25.4^{\#}$ \\
TAA+LES & $65.8 \pm 7.2^{*}$ & $127.2 \pm 13.9^{*}$ \\
TAA+LEC & $94.6 \pm 10.8^{* *}$ & $159.5 \pm 18.3^{* \#}$ \\
TAA+SIL & $76.5 \pm 8.5^{* \#}$ & $145.3 \pm 15.1^{* \#}$ \\
TAA+AMB & $96.6 \pm 10.2^{* \#}$ & $171.4 \pm 16.4^{* \#}$ \\
\hline
\end{tabular}

The data were stated as mean \pm SD. Means with different superscripts are significantly different. $* P<0.05$ versus TAA group and ${ }^{\#} P<0.05$ versus TAA+LES group. LES stands for Lesimarin. TAA stands for thioacetamide. LEC, SIL, AMB stand for lecithin, silymarin, Artemisia messerschmitiana Besser, respectively.

showed more remarkable effect on albumin level and $\mathrm{A} / \mathrm{G}$ ratio compared with those of individual constituents. Reduced level of albumin usually shows the severity of the condition, and it is a reliable index of the prognosis. Generally, the continuation of hypoalbuminaemia indicates a progress or a change to a chronic state (Thapa, Walia, 2007). The significant improvement at the level of biochemical parameters is indicative of the beneficial effects of Lesimarin in protecting the liver.

Grossly, the liver surfaces of the TAA group showed many spots of nodules, relatively harder in comparison with the normal gross features of the livers from the control (Figure 1A, 1B). In contrast to this, the livers from TAA+Lesimarin group were with much fewer nodular spots, softer in consistency, and with a comparatively normal liver size than the TAA group although the liver

TABLE III - Effect of TAA, lesimarin and its constituents on GGT, ALP activities and bilirubin level

\begin{tabular}{lccc}
\hline Group & GGT (U/L) & ALP (U/L) & Bilirubin(mg/dL) \\
\hline Control & $1.23 \pm 0.46^{* \#}$ & $152.1 \pm 18.9^{* \#}$ & $0.48 \pm 0.10^{*}$ \\
LES & $1.30 \pm 0.41^{* \#}$ & $147.2 \pm 15.4^{* \#}$ & $0.45 \pm 0.08^{*}$ \\
TAA & $8.32 \pm 2.67^{*}$ & $318.2 \pm 31.9^{\#}$ & $1.27 \pm 0.33^{\#}$ \\
TAA+LES & $3.13 \pm 1.76^{*}$ & $197.2 \pm 20.3^{*}$ & $0.59 \pm 0.15^{*}$ \\
TAA+LEC & $5.82 \pm 2.42^{* \#}$ & $241.3 \pm 23.7^{* \#}$ & $0.91 \pm 0.23^{* *}$ \\
TAA+SIL & $5.23 \pm 2.05^{* \#}$ & $232.5 \pm 21.6^{* \#}$ & $0.79 \pm 0.18^{* *}$ \\
TAA+AMB & $4.77 \pm 1.86^{* \#}$ & $226.6 \pm 19.8^{* \#}$ & $0.84 \pm 0.26^{* *}$ \\
\hline
\end{tabular}

The data were stated as mean $\pm \mathrm{SD}$. Means with different superscripts are significantly different. $* P<0.05$ versus TAA group and ${ }^{\#} P<0.05$ versus TAA+LES group. GGT stands for $\gamma$-glutamyltransferase. ALP stands for alkaline phosphatase.LES stands for Lesimarin. TAA stands for thioacetamide. LEC, SIL, AMB stand for lecithin, silymarin, Artemisia messerschmitiana Besser, respectively. 
TABLE IV - Effect of TAA, lesimarin and its constituents on total protein, albumin and A/G ratio

\begin{tabular}{lccc}
\hline Group & T-protein $(\mathbf{g} / \mathbf{d L})$ & Albumin $(\mathbf{g} / \mathbf{d L})$ & A/G \\
\hline Control & $7.35 \pm 0.46$ & $4.02 \pm 0.49^{*}$ & $1.20 \pm 0.19^{* \#}$ \\
LES & $7.38 \pm 0.43$ & $3.99 \pm 0.44^{*}$ & $1.17 \pm 0.18^{* \#}$ \\
TAA & $7.09 \pm 0.28$ & $1.89 \pm 0.26^{\#}$ & $0.36 \pm 0.08^{\#}$ \\
TAA+LES & $7.29 \pm 0.41$ & $3.68 \pm 0.38^{*}$ & $0.96 \pm 0.13^{*}$ \\
TAA+LEC & $7.20 \pm 0.35$ & $2.78 \pm 0.37^{* \#}$ & $0.65 \pm 0.10^{* \#}$ \\
TAA+SIL & $7.27 \pm 0.38$ & $3.15 \pm 0.32^{* \#}$ & $0.81 \pm 0.11^{* \#}$ \\
TAA+AMB & $7.16 \pm 0.33$ & $2.45 \pm 0.28^{* \#}$ & $0.52 \pm 0.09^{* \#}$ \\
\hline
\end{tabular}

The data were stated as mean $\pm \mathrm{SD}$. Means with different superscripts are significantly different. ${ }^{*} P<0.05$ versus TAA group and ${ }^{\#} P<0.05$ versus TAA+LES group. A/G stands for the ratio of albumin to globulin. LES stands for lesimarin. TAA stands for thioacetamide. LEC, SIL, AMB stand for lecithin, silymarin, Artemisia messerschmitiana Besser, respectively.
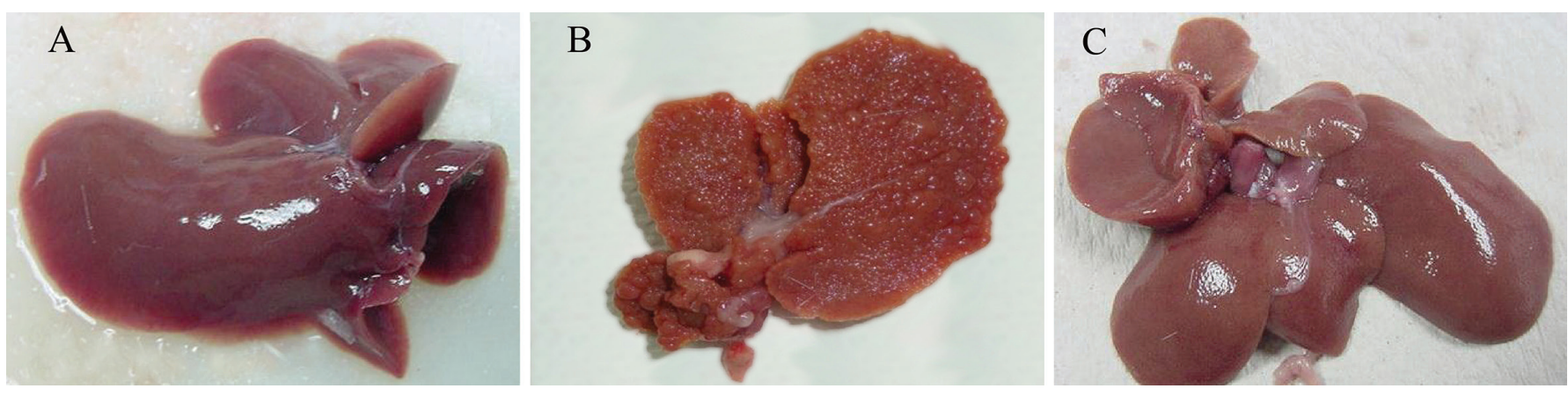

FIGURE 1 - Photographs showing the macroscopic appearances of livers from different groups: (A) control group-showing regular smooth surface, (B) TAA group-showing liver with multiple cirrhotic nodules on the whole surface of the liver, (C) TAA+Lesimarinshowing liver similar to normal with a smooth surface.

could not restore the deep red color of the normal control group (Figure 1C).

The appearance of HE-stained liver following 12 weeks of TAA and the result of lesimarin treatments are shown in Figure 2. Microscopic examinations in the present study showed that the severe liver damage induced by TAA could be significantly reduced by the administration of lesimarin. Livers from TAA-treated groups showed loss
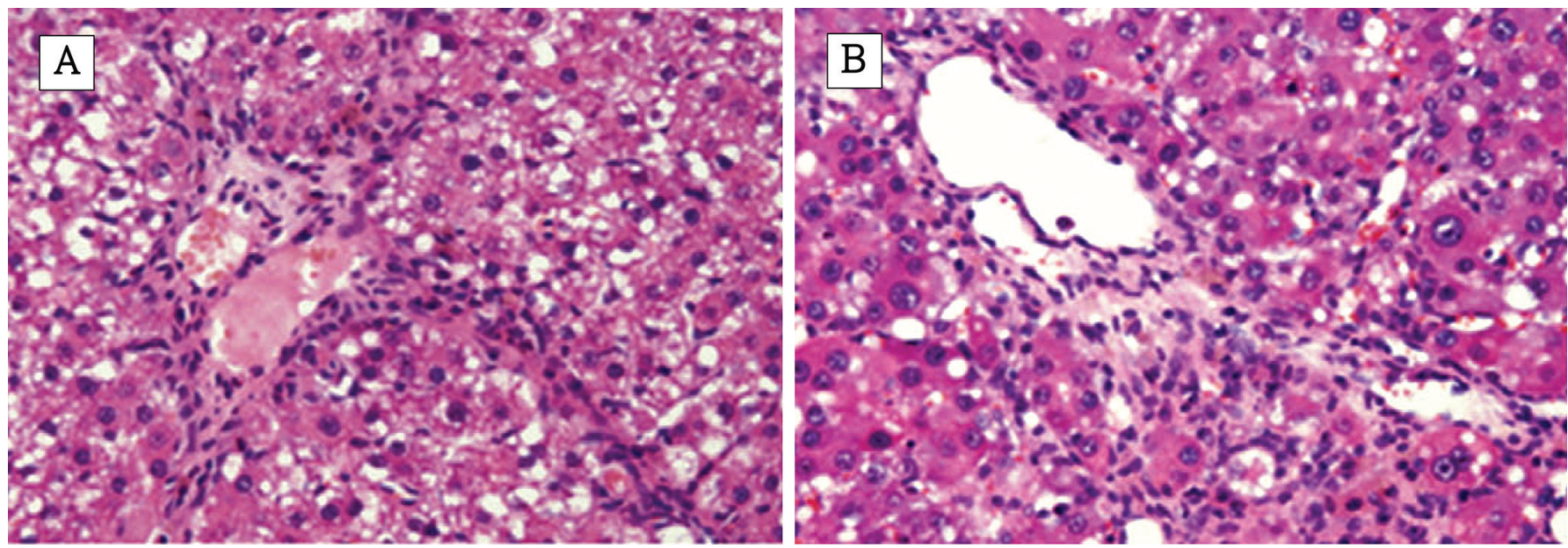

FIGURE 2 - Pictures showing the histopathological images of livers from different experimental groups (A)TAA group-showing marked vacuolar degeneration and fibrosis (B)TAA+LES group-showing mild vacuolation. Hematoxylin-Eosin stain. Magnification $\times 200$. 
of normal architecture with the presence of regenerating nodules separated by prominent fibrous septa extending from the central vein and marked vacuolar degeneration (Figure 2A). Compared with the lesions observed in the TAA group, the lesions of the Lesimarin-treated rats were of a much milder degree. These animals showed slight to moderate diffuse necrosis and vacuolations of hepatocytes (Figure 2B). This is in correlation with the results of the liver functional parameters on lesimarin-treated group.

On the other hand, TAA-treated rats showed an increase in hepatic levels of hydroxyproline. Oral administration of lesimarin for 12 weeks produced a significant reduction in hepatic levels of hydroxyproline in TAA-treated rats (Figure 3).
And there was remarkable reduction in the extent of fibrosis, which is probably due to reduced amount of stellate cells infiltration in rats treated with Lesimarin compared to TAA group microscopically. By using Masson's trichrome stain, it was found that the nodules of hepatocytes were separated only by thin fibrous septa in the lesimarin-treated group, in contrast by thick and remarkable fibrous septa in TAA group (Figure 4).

The improvement of TAA-induced hepatic fibrosis by lesimarin might be related to the lowering of fibrotic parameters such as hydroxyproline. Hydroxyproline is a common non-proteinogenic amino acid (Ostergaard, Holm, 1997). It is found only in collagen and elastin in mammals. Hydroxyproline is formed only by a post-

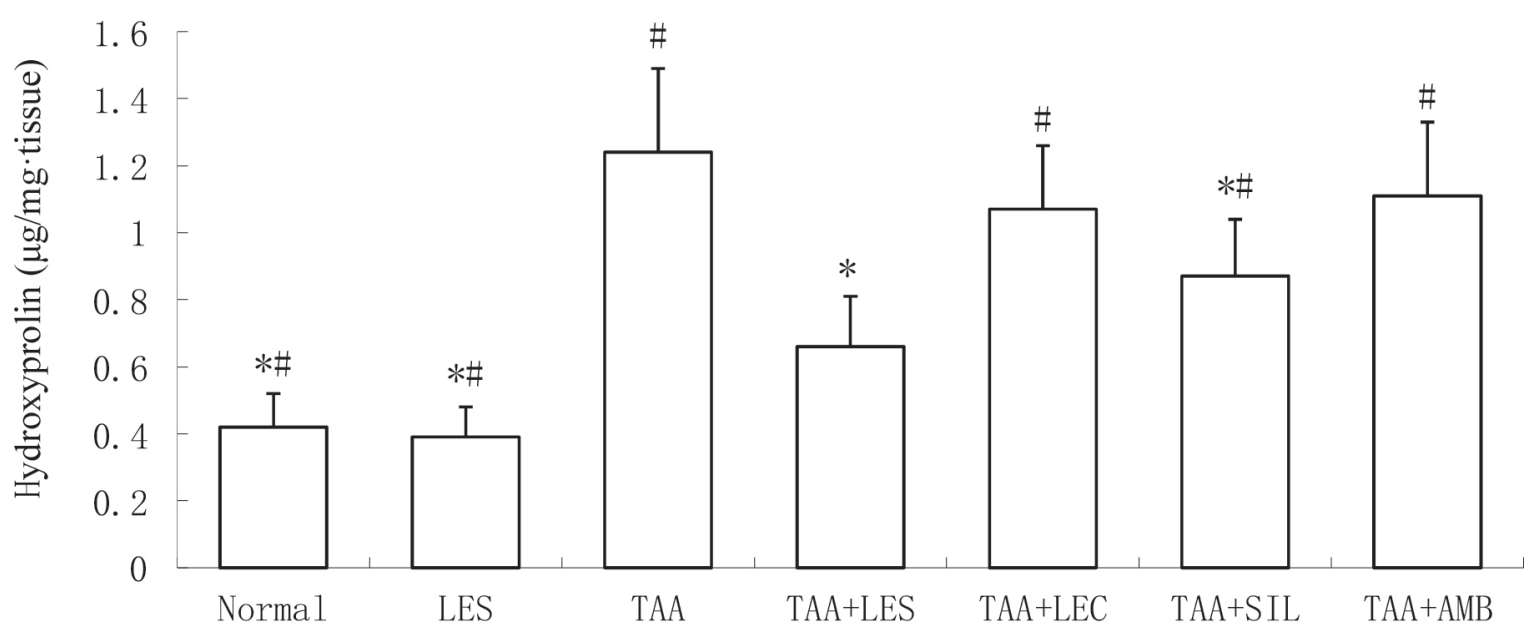

FIGURE 3 - Effect of TAA, lesimarin and its constituents on hydroxyprolin level. The data were stated as mean \pm SD. Means with different superscripts are significantly different. $* \mathrm{p}<0.05$ versus TAA group and $\# \mathrm{p}<0.05$ versus TAA+LES group. LES stands for Lesimarin. TAA stands for thioacetamide. LEC, SIL, AMB stand for lecithin, silymarin, Artemisia messerschmitiana Besser, respectively.
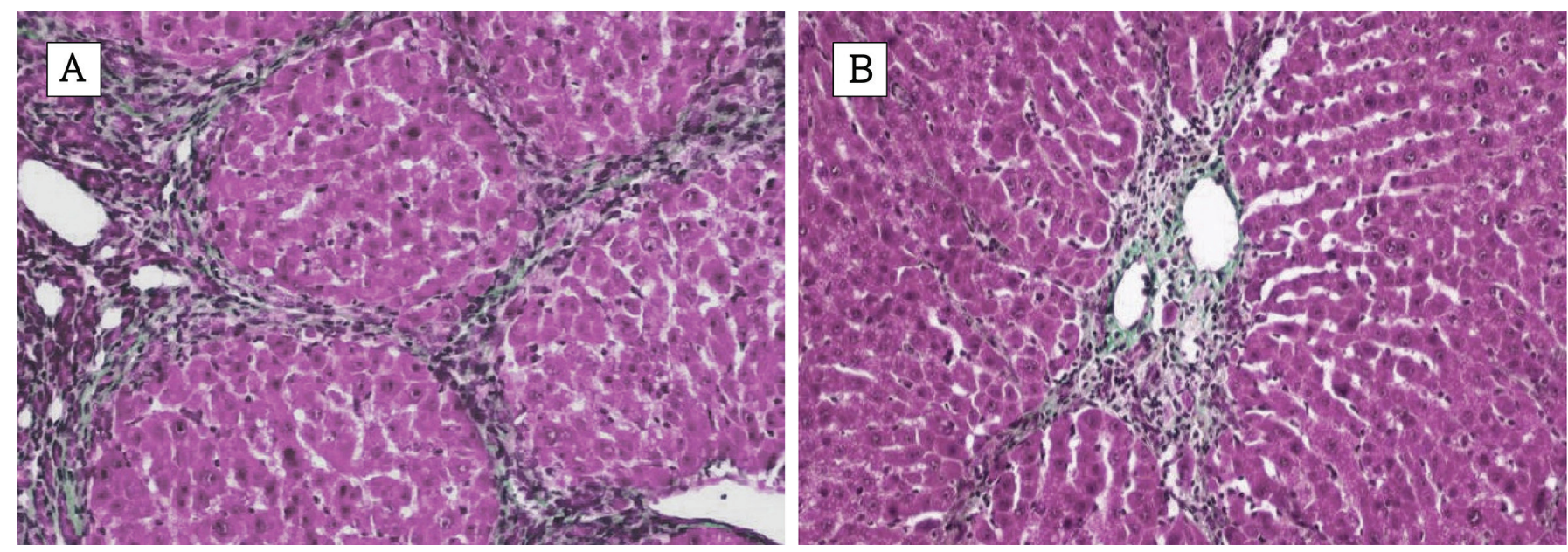

FIGURE 4 - Pictures showing the histopathological images of livers from different experimental groups: (A)TAA group-showing epithelial proliferation of bile duct and thick fibrous septa, (B)TAA+LES group-showing thin fibrous septa and mild epithelial proliferation of bile duct, Masson's trichrome stain. Magnification $\times 200$. 
translational modification in the peptide chain, and proline hydroxylase does not hydroxylate free proline. Hydroxyproline levels in tissue hydrolysates are a direct measure of the amount of collagen or gelatin present (Colgrave et al., 2012). A variety of disease states are believed to affect collagen turnover and can cause elevated serum or urine hydroxyproline levels. Such conditions include neoplastic, inflammatory, hepatic and autoimmune disorders. Thus, a reduction of hepatic hydroxyproline was considered for the attenuation of hepatic fibrosis (Li et al., 2011). In our results, lesimarin and silymarin lowered the hepatic hydroxyproline level in TAA-induced hepatic fibrosis showing an attenuation of hepatic fibrosis. And the other ingredients of mixture showed no significant improvement on hydroxyproline level compared with TAA group (Figure 3). That means silymarin is much more important than the rest constituents of the mixture in preventing liver fibrosis.

All our findings indicated lesimarin might be a protective agent against liver diseases such as liver cirrhosis. The injured liver cells stimulate hepatic stellate cells to transform into myofibroblast-like cells, which secrete large amount of collagen, thereby producing liver fibrosis. Activation of HSCs is also regarded as a major cause of liver fibrosis. HSC activation generates the alpha smooth muscle actin ( $\alpha$-SMA)-positive myofibroblastlike cells that are responsible for scar tissue formation in the fibrotic liver. On the other hand, the induction of free radical generation, mitochondrial dysfunction and depletion of antioxidants are effective in the progression of fibrosis and cirrhosis (Aydin et al., 2010; Chen et al., 2012).

A previous acute toxicity study showed nontoxic property of lesimarin, and the $\mathrm{LD}_{50}$ dose recorded was over $20 \mathrm{~g} / \mathrm{kg}$ body weight. In addition, there were no histological changes in liver, heart, and kidney in any of the doses of the lesimarin. This is consistent with our findings on the various parameters as shown in tables and figures.

Silymarin, a constituent of mixture, causes downregulation of MMPs (matrix metalloproteinases), TGF$\beta 1$ (transforming growth factor beta 1 ), $\alpha$-SMA(alpha smooth muscle actin) and COL- $\alpha 1$ (collagen alpha 1) on TAA-induced chronic liver fibrosis. MMPs are zincand calcium-dependent proteases that participate in the degradation of extracellular matrix (ECM) molecules. Major components of the ECM in liver fibrosis include collagen types I, III and IV. MMP-2, MMP-8, MMP-9 and MMP-13 cause fibrinolysis and tissue remodelling, which results in the progression of liver fibrosis. And theses enzymes are expressed on the HSCs. TAA-induced liver damage observed in the present study may be due to
MMP up-regulation and HSCs activation (Visse, Nagase, 2003). Such an activation and proliferation of HSCs which promote cell proliferation, collagen deposition and fibrogenesis is also inhibited by silymarin administration (Abenavoli et al., 2011; Michaela, Gerry, Miles, 2015; Sario et al., 2005). In addition, it is well known to be safe in animal models and possess potent antioxidant and hepatoprotective properties by contributing to reduced lipid peroxidation, increased liver glutathione level and stabilization of the membrane permeability (Abouzeinab, 2013; Chen et al., 2012).

Lecithin is an important component of cell membranes and forms ultrastructure of mitochondria and microsomes and is recognized as an indispensable substance for the maintenance of various enzyme activities. Lecithin is related to the architecture of membranes and microgranules of the hepatic cells and membrane permeability and the enzyme activities. It is also associated with the energy-producing system and oxidative phosphorylation in the mitochondria. Some researchers have proved that phopholipid-containing drugs are effective in the treatment of liver diseases. Lecithin also improves lipid metabolism in the liver and prevents the exacerbation of liver diseases. It has been used as a matrix to improve the lipophilic property and targeted delivery of bioactive compounds. It has been reported that silymarin has poor water solubility and bioavailability, but lecithin makes it 10 times more bioavailable (Korbanjhon, Chungang, 2013; Michaela, Gerry, Miles, 2015). This property of lecithin appears to contribute to the better effect of mixture than individual constituents.

Besides Artemisia messerschmidtiana Besser extract has remarkable cholagogic action, thereby showing positive action against the diseases of liver and biliary tract. It is well known to decrease AST and ALT activities in the various animal models associated with liver diseases. Lecithin and Artemisia messerschmidtiana Besser extract are supposed to help the hepatoprotective action of silymarin through their actions on the liver.

\section{CONCLUSIONS}

From the above observations, it can be concluded that lesimarin was effective in attenuating liver fibrosis induced by TAA as evidenced by histological and biochemical analyses. It is shown to have more potent hepatoprotective effect as compared with its individual constituents, that suggests the superiority of combination. Accordingly, this drug could be used as an effective product for the prevention and treatment of chronic liver diseases including hepatic cirrhosis. 


\section{ACKNOWLEDGMENTS}

We would like to thank Dr. Hong Nam J. for the preparation and determination of histological data and appreciate Mr. Song Chan H. for skilled technical assistance in this research.

\section{REFERENCES}

Abenavoli L. Role of silymarin to treat fibrosis development in non-alcoholic fatty liver disease. Hepatol Res. 2011;41(7):668.

Abouzeinab NS. Cytoprotectve effect and antioxidant properties of silymarin on cisplatin induced hepatotoxicity in rats: a biochemical and histochemical study. Int J Cancer Res. 2013;9(1):9-23.

Abul H, Mathew TC, Dashti HM, Al-Bader A. Level of superoxide dismutase, glutathione peroxidase and uric acid in thioacetamide-induced cirrhotic rats. Anat Histol Embryol. 2002;31(2):66-71.

Adeyemo O, Doi H, Rajender Reddy K, Kaplan DE. Impact of oral silymarin on virus- and non-virus-specific T-cell responses in chronic hepatitis C infection. J Viral Hepat. 2013;20(7):453462.

Aydin AF, Küskü-Kiraz Z, Doğru-Abbasoğlu S, Güllüoğlu M, Uysal M, Koçak-Toker N. Effect of carnosine against thioacetamide-induced liver cirrhosis in rat. Peptides. 2010;31(1):67-71.

Bataller R, Brenner DA. Liver fibrosis. J Clin Invest. 2005;115(2):209-218.

Benkoel L, Chanussot F, Dodero F, De la Maisonneuve C, Bongrand P, Benoliel AM, et al. effect of dietary lipids (soybean lecithin and triacylglycerol) on hepatic F-actin microfilaments in cyclosporine A-treated rats. Dig Dis Sci. 2000;45(6):1096-1102.

Carina PC, Rodrigo PC, Antonio SM, Renato PC, Nair SY, Adelar B, et al. Metabolic effects of silibinin in the rat liver. Chem Biol Interact. 2012;195(2):119-132.

Chen IS, Chen YC, Chou CH, Chuang RF, Sheen LY, Chiu CH. Hepatoprotection of silymarin against thioacetamide-induced chronic liver fibrosis. J Sci Food Agric. 2012;92(7):1441-1447.
Chilakapati J, Korrapati MC, Hill RA, Warbritton A, Latendresse JR, Mehendale HM. Toxicokinetics and toxicology of thioacetamide sulfoxide: a metabolite of thioacetamide. Toxicology. 2007;230(2-3):105-106.

Ching Yang Chi, Yi Fang Jong, Liang Hong Tuan, Ching Wang Tzu, En Zhou Ya, Chen Lin Ta. Potential antioxidant properties and hepatoprotective effects of an aqueous extract formula derived from three Chinese medicinal herbs against CCl4-induced liver injury in rats. Int Immunopharmacol. 2013;15(1):106-113.

Chung-Hsi Chou, Yi-Chen Chen, Meng-Chien Hsu, Wei-Lun Tsai, Chia-Yi Chang, Chin-Hsien Chiu. Effect of silymarin on lipid and alcohol metabolism in mice following long-term alcohol consumption. J Food Biochem. 2012;36(3):369-377.

Colgrave ML, Allingham PG, Tyrrell K, Jones A. Multiple reaction monitoring for the accurate quantification of amino acids: using hydroxyproline to estimate collagen content. Methods Mol Biol. 2012;828:291-303.

Freedman ND, Curto TM, Morishima C, Seeff LB, Goodman $\mathrm{ZD}$, Wright EC, et al. Silymarin use and liver disease progression in the hepatitis $\mathrm{C}$ antiviral long-term treatment against cirrhosis trial. Aliment Pharmacol Ther. 2011;33(1):127-137.

Konovalova OA, Rybalko KS. Coumarins of species of the genus Artemisia [messerschmidtiana, freyniana. Chem-NatCompd. 1977;12(1):86.

Korbanjhon B, Chungang C. physicochemical properties of diosmetin and lecithin complex. Trop J Pharm Res. 2013;12(4):453-456.

Laleman W, Vander Elst I, Zeegers M. A stable model of cirrhotic portal hypertension in the rat: thioacetamide revisited. Eur J Clin Invest. 2006;36(4):242-249.

Li W, Zhu C, Chen X, Li Y, Gao R, Wu Q. Pokeweed antiviral protein down-regulates $\mathrm{Wnt} /$ beta-catenin signalling to attenuate liver fibrogenesis in vitro and in vivo. Dig Liver Dis. 2011;43(7):559-566.

Ljubuncic P, Song H, Cogan U, Azaizeh H, Bomzon A. The effects of aqueous extracts prepared from the leaves of Pistacia lentiscus in experimental liver disease. J Ethnopharmacol. 2005;100(1-2):198-204. 
Madani H, Talebolhosseini M, Asgary S, Naderi GH. Hepatoprotective activity of silybum marianum and cichorium intybus against thioacetamide in rat. Pak J Nutrit. 2008;7(1):172176.

Mahmood R, Javed I, Arif M, Hafiza SR, Muhammad SQ, Muhammad A, et al. Hepatoprotective effects of silybum marianum (Silymarin) and glycyrrhiza glabra(Glycyrrhizin) in combination: a possible synergy. Evid-Based Complement Alternat Med. 2014;2014:641597.

Maida D, Sugas S, Marica B. Phosphatidylcholine functional foods and nutraceuticals: A potential approach to prevent non-alcoholic fatty liver disease. Eur J Lipid Sci Technol. 2012;114(4):389-398.

Michaela P, Gerry M, Miles F. Silymarin. Pract Diabetes. 2015;32(4):148-150.

Ostergaard S, Holm A. Synthesis and screening of an indexed motif-library containing non-proteinogenic amino acids. J Pept Sci. 1997;3(2):123-132.

Palacios RS, Roderfeld M, Hemmann S, Rath T, Atanasova $\mathrm{S}$, Tschuschner A, et al. Activation of hepatic stellate cells is associated with cytokine expression in thioacetamide-induced hepatic fibrosis in mice. Lab Invest. 2008;88(11):1192-1203.
Sario AD, Bendia E, Taffetani S, Omenetti A, Candelaresi C, Marzioni M, et al. Hepatoprotective and antifibrotic effect of a new silybin-phosphatidylcholine-Vitamin E complex in rats. Dig Liver Dis. 2005;37(11):869-876.

Shaker ME, Salem HA, Shiha GE, Ibrahim TM. Nilotinib counteracts thioacetamide-induced hepatic oxidative stress and attenuates liver fibrosis progression. Fundam Clin Pharmacol. 2011;25(2):248-257

Switzer BR. Determination of hydroxyproline in tissue. J Nutr Biochem. 1991;2:229-231.

Thapa BR, Walia A. Liver function tests and their interpretation. Indian J Pediatr. 2007;74(7):663-671.

Visse R, Nagase H. Matrix metalloproteinases and tissue inhibitors of metalloproteinases: structure, function, and biochemistry. Circ Res. 2003;92(8):827-839.

Received for publication on $22^{\text {nd }}$ January 2018 Accepted for publication on $03^{\text {rd }}$ August 2018 